\title{
RheinGAU 2001
}

\author{
Am 31. März dieses Jahres veranstalteten die Regional- \\ gruppen Rheinland-Pfalz, Saarland und Hessen des Ärzte- \\ verbandes Deutscher Allergologen ihre Jahrestagung im \\ Rahmen der Rheinischen Gespräche zu Allergologie und \\ Umweltmedizin. Vier der Referenten fassen hier ihre \\ Forschungsergebnisse zusammen.
}

\section{Die aktuelle Hygiene-Hypothese}

M ehrere Autoren haben eine starke inverse Korrelation zwischen der Anzahl der Geschwister und Heuschnupfen bei Kindern und Jugendlichen aufgezeigt. Ältere Geschwister hatten einen stärkeren protektiven Effekt als jüngere. Daraus wird gefolgert, dass häufige virale und bakterielle Infekte der oberen Atemwege im Kindesalter atopische Erkrankungen möglicherweise vorbeugen. Diese Hypothese ist zunehmend bestätigt worden. Der frühe Krippen-Eintritt in den neuen Bundesländern war negativ mit der AllergiePrävalenz korreliert. Eine aktuelle Studie aus den USA ergab ebenfalls einen inversen Zusammenhang mit frühem Krippen-Eintritt und der Entwicklung von Asthma bronchiale bis zum Adoleszentenalter. Die Deutsche Multizentrische Geburtskohortenstudie zeigte ferner, dass Kinder, die im ersten Lebensjahr häufig Schnupfen hatten, bis zum Schulalter seltener Asthma entwickelten. In einer Querschnittsstudie mit italienischen Rekruten waren positive Hepatitis-A- oder Toxoplasma-gondiiSerologien negativ mit allergischen Erkrankungen korreliert.

\section{Primärprävention auf dem Bauernhof}

Aktuelle Studien zeigten, dass Bauernkinder im Vergleich zu Kindern, die im selben Dorf, aber nicht auf dem Bauernhof aufwachsen, wesentlich seltener an Allergien leiden. Lebensbedingungen auf einem Bauernhof unterscheiden sich von denen anderer Familien: Bauernfamilien sind im Schnitt größer, sie halten häufiger Katzen und Hunde die aber in der Regel draußen leben und keinen Zugang zum Bett der Kinder haben. In Bauernhäusern wird häufiger noch mit Holz und Kohle geheizt und die Mütter rauchen im Schnitt seltener. Auch unterscheiden sich die Ernährungsgewohnheiten von Bauern- und anderen Familien. Es ist derzeit noch unklar, welche Lebenstilfaktoren diesen protektiven Effekt im einzelnen ausmachen. Erste Befunde deuten aber darauf hin, dass der Aufenthalt im Stall und der Konsum von Rohmilch und damit die mikrobielle Belastung ein wesentlicher Erklärungsfaktor sein könnte.

\author{
PD Dr. Erika von Mutius \\ Dr. v. Haunersche Kinderklinik der \\ Universität München
}

\section{Anti-lgE-Antikörper gegen Asthma}

D ei der großen Mehrzahl der Patienten mit Asthma bronchiale liegt der Erkrankung eine allergisch ausgelöste chronische Entzündung der Atemwege zu Grunde. Dem IgE-Antikörper kommt eine zentrale Rolle zu, indem er zellgebunden das relevante Antigen erkennt und damit Mastzellen und Basophile aktiviert. Diese Zellaktivierung ist einer der Hauptmechanismen für die Unterhaltung der chronischen eosinophilen Entzündung der respiratorischen Schleimhäute. Während die heutigen anti-inflammatorisch ausgerichteten Therapien eine unspezifische Unterdrückung dieser Entzündungsreaktion anstreben, hat der innovative Ansatz einer Anti-IgE-Therapie zum Ziel, unter Ausnützung der Schlüsselposition des $\mathrm{IgE}$ in der allergischen Kaskade eine der wesentlichen Ursachen dieser chronischen Entzündung zu beeinflussen.
Den Firmen Genentec und Novartis ist die Entwicklung eines Anti-IgEAntikörpers gelungen, der inzwischen in großen klinischen Studien erfolgreich getestet wurde. Grundlage dieses Therapie-Konzeptes ist eine spezifische Bindung aller IgE-Moleküle, unabhängig von ihrer Antigen-Spezifität, in einer Weise, die nicht zur Mastzell-Aktivierung führt. Dabei wurde ein monoklonaler muriner Antikörper gefunden, der die IgE-Antikörper am Fc-Segment bindet, in einer Region, die üblicherweise vom hochaffinen IgE-Rezeptor auf Mastzellen und Basophilen als Bindungsstelle benutzt wird. Der murine Antikörper wurde in der Folge molekularbiologisch derart mit einem humanen IgG-kappa-Molekül verknüpft, dass lediglich ein ca. 5\%iger Anteil der murinen Aminosäure-Sequenz, nämlich die unmittelbar die Bindungsstelle 